\title{
Estudio Inferencial en el desarrollo de Competencias Científicas que orientan los docentes en Básica Primaria
}

\section{Inferential study in the development of scientific competences oriented by elementary teachers.}

\section{Estudo Inferencial no desenvolvimento de Competências Científicas para orientar os professores do ensino fundamental}

\author{
Alba Yuli Marulanda-Echeverri ${ }^{1}$
}

Forma de citar: Marulanda-Echeverri, A. Estudio Inferencial en el desarrollo de Competencias Científicas que orientan los docentes en Básica Primaria, Revista Eco.Mat. 7 [92-100].

Recibido:

Mayo 7 de 2015

Aceptado:

Agosto 3 de 2015

\section{Resumen}

El propósito de la investigación es caracterizar las prácticas pedagógicas que se utilizan en el desarrollo de competencias científicas en educación básica primaria. Así mismo, surge la inquietud de crear actividades didácticas que fortalezcan las competencias científicas tendientes a mejorar el desempeño por parte de los estudiantes a través de la práctica pedagógica. El diseño de la investigación se realiza bajo el paradigma cuantitativo apoyado en análisis cualitativo, es de Campo; los datos son recopilados en forma directa a través de encuesta escala tipo Likert con preguntas dirigidas de los estándares establecidos por el Ministerio de Educación Nacional, organizadas por Competencias Genéricas, Competencias Docentes y Competencias científicas; con una fiabilidad del $(0,87)$, y un R de $(0,93)$. En los hallazgos, Se pudo observar en el semáforo amarillo que aún falta un cambio en la aplicación de las competencias específicas y genéricas por falta de mayor entrega de los docentes; que deberían generar aprendizajes socialmente significativos, tener dominio y destrezas para enseñar y desarrollar habilidades de pensamiento en los niños, situar su práctica pedagógica de acuerdo a las condiciones del contexto del educando.

Palabras clave: Ciencias Naturales, Competencias, Educación Básica Primaria, Práctica Pedagógica.
Abstract
The purpose of the research is to characterize the pedagogical practices that are used in the development of scientific competences in elementary education. Likewise, a concern arise in order to create didactic activities that strengthen the scientific competences tending to improve the student's performance through pedagogical practice. The design of the research is done under the quantitative paradigm supported by qualitative analysis, it is a field study where data are collected directly through a Likert scale survey with questions directed from the standards established by the MEN, organized by Generic Competences, Teaching Competences and Scientific Competences; with a reliability of (0.87), and an R of (0.93). It was observed in the yellow traffic light that a change in the application of specific and generic competences is still missing, due to a lack of engagement from the teachers, who must generate socially meaningful learning, have mastery and skills to teach and develop thinking 
skills in children, and locate their pedagogical practice according to the conditions of the context of the student.

Keywords: natural sciences, skills, primary basic education, pedagogical practice.
Enero-Diciembre 2016 ISSN 1794-8231

E-ISSN 2462 - 8794 PP: 92-100

\section{Resumo}

O propósito da pesquisa é caracterizar as práticas pedagógicas que se utilizam no desenvolvimento de competências científicas no ensino fundamental. Assim mesmo, surge a inquietude de criar atividades didáticas que fortaleçam as competências científicas tendentes a melhorar o desempenho por parte dos estudantes a través da prática pedagógica. $\mathrm{O}$ delineamento da pesquisa se realiza sob o paradigma quantitativo apoiado em análise qualitativo, é de Campo; os dados são recopilados em forma direta a través de levantamentos a escala tipo Likert com preguntas dirigidas dos estândares estabelecidos pelo Ministério da Educação Nacional, organizadas por Competências Genéricas, Habilidades de Ensino e Competências Científicas; com uma fiabilidade do 0,87, e um R de 0,93 . Nos resultados verificou-se no semáforo amarelo que ainda falta uma mudança na aplicação das competências específicas e genéricas por falta de maior compromisso dos professores; que deveriam gerar aprendizagens socialmente significativos, ter domínio e habilidades de ensino e desenvolver destrezas de pensamento em crianças, colocando a sua prática pedagógica de acordo às condições do contexto do aprendiz.

Palavras-chaves: ciências naturais, competências, ensino fundamental, prática pedagógica.

\section{Introducción}

Cada Institución Educativa tiene un propósito fundamental de formación integral en sus estudiantes, enmarcado dentro de su proyecto educativo institucional y su modelo pedagógico, para ser reconocida por la alta calidad en la formación de jóvenes con sentido de responsabilidad social y la generación de conocimientos que implican una mejor calidad de vida profesional. Es importante que conozcamos, que, en el nivel de educación primaria, las competencias científicas llevan inmersas otras competencias, que son relevantes para la formación de los estudiantes, ya que se espera, sean referentes de calidad, para dar paso al nivel de bachillerato, arrojando un buen resultado en sus pruebas de SABER, por la implementación de estrategias de mejoramiento por parte de sus docentes, donde se aplican las herramientas apropiadas para conducir los educandos por el camino de un aprendizaje lógico y adecuado durante las prácticas pedagógicas; (aprendizaje significativo).

La introducción que la Ley General de Educación de Colombia hace en el currículo escolar respecto al término "competencias básicas" no es una mera definición de un concepto, sino que implica una completa reformulación de los métodos de enseñanza. Del "saber" al "saber hacer", de "aprender" a "aprender a aprender"; y el más reciente aprender a convivir, en donde vienen inmersas las competencias ciudadanas. El objetivo es que una vez cumplida la etapa de escolarización obligatoria, los jóvenes hayan alcanzado una serie de competencias que les permitan desenvolverse en su vida adulta y en el mercado laboral de manera satisfactoria; a través de los saberes previos 
que se complementan con el aprendizaje significativo, pero esto implica que los profesores cambien su manera de instruir y estimular el proceso de aprendizaje de los alumnos, a través de sus asignaturas.

Es necesario partir desde la base, para que los términos que operan los componentes dinamizadores en cuanto a la ejecución de las estrategias mencionadas, por tratarse de una temática debatida en el ámbito de la educación; tomando las competencias como un macro, por tratarse de una gran diversidad de variables que se asumen de acuerdo al contexto escolar; las cuales se sitúan dentro del grupo, competencias científicas, y las prácticas pedagógicas. Para ello, es importante hacer un barrido bibliográfico en cuanto a conceptos y autores que las enmarcan en el sentido de responsabilidad social y la generación de conocimiento.

\section{Práctica Pedagógica}

El aprendizaje del educador al educar se verifica en la medida que el educador humilde $\mathrm{y}$ abierto se encuentre permanentemente disponible para repensar lo pensado, revisar sus posiciones; en que busca involucrarse con la curiosidad del alumno y los diferentes caminos y senderos que ella lo hace reconocer. (Freire, 1997, p, 28).

Según Martínez Boom (2004) la práctica pedagógica da cuenta de tres aspectos relevantes en cuanto a la pedagogía como parte de un todo como lo son la práctica, el saber y la disciplina.

Como se plantea, el secreto está en lo que los profesores comprenden: su asignatura y valoran el aprendizaje humano en este caso el educando. Un buen profesor conoce su materia a fondo, pero esto no es lo más importante para él; es causar en los escolares una tracción enlazada en respuestas significativas. (Bain Ken 2006)
Se deben generar competencias primordiales, producir ero pedagogía (enseñanza con pasión), ser profesores apasionados por lo que se enseña para que la respuesta sea devuelta con el efecto esperado, con el potencial oculto que cada educando posee. (Urbina 2010)

En conclusión; en cada educador debe existir una evaluación constante de su quehacer pedagógico que lo enriquezca en sus prácticas pedagógicas, así como la realización de una pedagogía crítica que se vea reflejada en las clases cotidianas de sus alumnos y su aprendizaje significativo donde se propenda por un currículo oculto y flexible, que se adopte en forma permanentemente al contexto de sus educandos.

\subsection{Competencias}

La competencia científica implica: "la capacidad de aplicar el conocimiento científico en situaciones de la vida real, lo que requiere conocer ciencia, los conceptos $\mathrm{y}$ las teorías fundamentales, y conocer, respecto de la ciencia, sus metodologías, su poder y sus limitaciones, su interrelación con la sociedad... Además, también requiere la disposición a ejercitar estas competencias, lo cual depende de las actitudes de los individuos hacia la ciencia y su disposición a implicarse en cuestiones relacionadas con las ciencias" (Según PISA, 2009).

El proyecto Tuning ha sido creado en Europa y desarrollado también en América Latina con el fin de encontrar puntos comunes de referencia que contribuyen a facilitar la cooperación académica entre los distintos países. Las competencias se constituyen en uno de esos ejes comunes, dado que permiten la articulación entre niveles y sistemas educativos, y, por consiguiente, facilitan la movilidad de los estudiantes y docentes, así como la comparación y el reconocimiento de títulos. (21-23 febrero, 2007, México)

Tuning ha definido las competencias como 
"una combinación dinámica de conocimiento, comprensión, capacidades y habilidades... "se forman unidades del curso y se evalúan en diferentes ciclos $y$ se dividen en competencias relacionadas con un área de estudio (específicas de un campo de estudio) y genéricas (comunes para diferentes cursos) y estas últimas permiten a los ciudadanos adaptarse a los desafíos de la presente sociedad que cada día debe tener un pensamiento flexible, saber interpretar, enfrentar y resolver las situaciones problemáticas y enfrentar las incertidumbres.

En este campo Tuning América Latina ha identificado 27 competencias genéricas, las cuales ha agrupado de acuerdo a su afinidad en cuatro factores: Proceso de Aprendizaje, valores Sociales, Contexto Tecnológico Internacional y Habilidades Interpersonales.

Teniendo como partida estas bases conceptuales, el docente ideal como ente dinamizador, a partir de la observación podrá empezar a trabajar las competencias en ciencias naturales en educación básica primaria, Proyecto DESECO (Definición y Selección de Competencias) de la OCDE, como "la capacidad de responder a demandas complejas y llevar a cabo tareas diversas de forma adecuada". La inclusión de las competencias básicas en el currículo exige a los docentes un gran esfuerzo en el cambio de las rutinas docentes y las prácticas de aula.

\subsection{Competencias en Ciencias Naturales o Científicas}

Los Estándares básicos de Competencias en Ciencias Naturales tienen un énfasis en competencias, para el desarrollo de las habilidades y actitudes científicas de los estudiantes. Para ello, los estándares recomiendan que se fomente en la educación en ciencias naturales la capacidad de:

- Explorar hechos y fenómenos.

- Analizar problemas.
- Observar, recoger y organizar información relevante.

- Utilizar diferentes métodos de análisis.

- Evaluar los métodos.

- Compartir los resultados.

Además, se busca con estos estándares, que en las instituciones educativas se creen espacios adecuados para "que el estudiante construya su aprendizaje frente a la investigación y que se aproxime al conocimiento a través de la búsqueda. Esto implica que aprenda a recoger datos fidedignos, analizarlos y encontrar relaciones entre ellos, aprender a comunicar lo que se ha descubierto; todo esto debe estar estrechamente ligado a los conocimientos que ya fueron establecidos en las Ciencias Naturales tales como la física, la química o la biología. Con esta aproximación como científico, el estudiante podrá llegar a tener compromisos sociales que se relacionan con las ciencias sociales y con las competencias ciudadanas" (Al tablero No 30, Pagina. 4).

En Desarrollo de competencias cientificas a través de la aplicación de estrategias didácticas alternativas. Un enfoque a través de la enseñanza de las Ciencias Naturales. Colombia. Consideran al docente como un profesional reflexivo, que es capaz de criticar su práctica pedagógica y transformar los criterios para la selección de los contenidos. (Torres, Mora, Garzón y Ceballos, 2013)

Cuando un niño se enfrenta a un fenómeno o un problema nuevo lo hace desde el sistema de conocimientos que ha podido construir hasta el momento. En La enseñanza de las Ciencias Naturales y educación ambiental en las instituciones educativas oficiales del departamento de Nariño. Colombia. (MEN 2004). Destacan en su investigación lo dicho por Husserl, E. donde para el "todo conocimiento proviene del Mundo de la Vida y tiene sentido sólo en él" (Torres y Barrios 2009). Estas consideraciones permiten comprender la importancia de seleccionar los
Enero-Diciembre 2016 ISSN 1794-8231 E-ISSN 2462 - 8794 PP: 92-100 
criterios, seleccionar los contenidos temáticos, para permitir que el estudiante relacione, lo que aprende en Ciencias Naturales, con las decisiones que toma en la vida cotidiana.

Se modificó la propuesta al hablar de estándares básicos de competencia y los definió como "criterios claros y públicos que permiten conocer lo que deben aprender nuestros niños, niñas y jóvenes, y establecer en el punto de referencia de lo que están en capacidad de saber y saber hacer en cada una de las áreas y los niveles" (MEN, 2004)

En la Evaluación por competencias y estándares de competencia en el campo de la enseñanza de las ciencias y la educación ambiental. En Colombia, se debe hacer énfasis en las necesidades de una evaluación por competencias y su articulación a la formación del profesorado, sustentadas en la innovación y la investigación docente en el aula (Mora y Parga, 2005).

Además, se menciona que ninguna innovación en la educación científica ha sido exitosa sin el soporte y la innovación de los profesores en las aulas, por lo cual es fundamental ayudar al profesorado a cambiar la percepción sobre la naturaleza de las ciencias, su enseñanza, su aprendizaje y su evaluación.

\section{Metodología}

El diseño de la investigación se realiza bajo el paradigma cuantitativo apoyado en análisis cualitativo, (Morse, 2003).

96 Según la particularidad propia que tiene la investigación es de Campo; los datos de interés son recopilados en forma directa a través de encuestas con preguntas dirigidas a los estándares establecidos por el MEN y organizados para un análisis y aplicación de una metodología sistemática de resolución de problemas (Arias ,2006). De la misma forma se asumió la investigación como un diseño descriptivo en la medición de variables de estudio.
Durante la etapa inicial de la investigación para la recolección de la información se adaptó y aplicó una encuesta, tipo escala Likert con opciones: 1 Nunca, 2 Algunas Veces, 3 Siempre. Organizadas por Competencias Genéricas, Competencia Docente y Competencias científicas; También se recoge información básica como género, edad e información socio académica. Se aplicó una prueba piloto, en una Institución Educativa diferente; el instrumento fue analizado por tres expertos y su fiabilidad fue de (alfa de Cronbach de 0,87), Un coeficiente de correlación $\mathrm{R}$ de 0.93 lo cual indica coherencia en los elementos del instrumento., se realizó un análisis factorial para reducción de ítems, antes de su aplicación final,

Se consideró importante elegir como participantes de muestreo, los grados tercero, cuarto y quinto de primaria; se excluyó los grados primero y segundo, por su dificultad para leer e interpretar los ítems del instrumento. Para cada uno de los grados se seleccionó estudiantes mediante muestreo aleatorio simple. El total de participantes quedó conformado por 193 estudiantes ambos sexos, de tres sedes de primaria cuya edad oscilaba entre los (8-14) años; al igual que 6 docentes del área, con edades entre los (21-62) años; con un 90\% de confianza; de esta forma se garantizó, que cada uno de los estudiantes matriculados en cada grado, tuviesen la misma probabilidad de selección.

A partir de la información inicial para cada instrumento se realizó análisis de tipo descriptivo buscando identificar las características etnográficas de los grupos, valoración de desempeño de los docentes con respecto a la opinión de sus estudiantes y encontrar las relaciones, entre profesionalización y competencias para hacer aportes conceptuales a los procesos pedagógicos dados por los docentes del área de ciencias naturales. 


\section{Resultados}

A continuación, se presentan los datos estadísticos correspondientes a las competencias en Ciencias Naturales o
Competencias Científicas en básica primaria desarrolladas en las prácticas pedagógicas, por los docentes de la Institución Educativa Julio Pérez Ferrero de la Ciudad de Cúcuta. (Tabla I).

Tabla I. Resumen. Competencias científicas en Ciencias Naturales

\begin{tabular}{|c|c|c|c|c|}
\hline & & Nunca & Aveces & Jamás \\
\hline \multicolumn{5}{|c|}{ COMPETENCIAS GENÉRICAS } \\
\hline Instrumental & Analiza los diferentes temas y los resume en forma sencilla para que los entiendas. & $5 \%$ & $12.1 \%$ & $82.9 \%$ \\
\hline Sistemática & Enseña a tener iniciativa en los proyectos, a luchar por las metas que se quieren alcanzar en la vida. & $3.1 \%$ & $11.9 \%$ & $85 \%$ \\
\hline Personales & Orienta en el aprendizaje de hacer las cosas bien y con agrado (compromiso ético). & $1 \%$ & $22.8 \%$ & $76.2 \%$ \\
\hline \multicolumn{5}{|c|}{ COMPETENCIA DOCENTE } \\
\hline \multirow{3}{*}{$\begin{array}{l}\text { Pedagógico } \\
\text { (formar) }\end{array}$} & Enseñas temas de actualidad & $1.6 \%$ & $34.2 \%$ & $64.2 \%$ \\
\hline & Diseña actividades teniendo en cuenta al estudiante & $7.3 \%$ & $32.1 \%$ & $60.6 \%$ \\
\hline & Utiliza el lenguaje apropiado con la comunidad educativa & $4.1 \%$ & $19.2 \%$ & $76.7 \%$ \\
\hline $\begin{array}{l}\text { Evaluación } \\
\text { (Mide el } \\
\text { Conocimiento) }\end{array}$ & $\begin{array}{l}\text { Contribuye a que despiertes la creatividad a partir de la presentación de algunos trabajos artísticos } \\
\text { y otros }\end{array}$ & $3.6 \%$ & $38.9 \%$ & $57.5 \%$ \\
\hline \multirow[t]{3}{*}{ Curricular } & Participa en capacitación & $3.6 \%$ & $21.2 \%$ & $75.1 \%$ \\
\hline & Promueve los derechos humanos & $3.6 \%$ & $17.1 \%$ & $79.3 \%$ \\
\hline & Fomenta el conocimiento científico a través de la investigación & $6.7 \%$ & $35.2 \%$ & $58 \%$ \\
\hline \multicolumn{5}{|c|}{ COMPETENCIA CIENTIFICA (interpreta, argumenta y propone) } \\
\hline \multirow[t]{3}{*}{ Entorno vivo } & Fomenta la higiene corporal & $6.2 \%$ & $29.5 \%$ & $64.2 \%$ \\
\hline & Explica las teorías del origen de la vida & $5.7 \%$ & $36.3 \%$ & $58 \%$ \\
\hline & explica la adaptación en los ecosistemas & $3.6 \%$ & $29 \%$ & $67.4 \%$ \\
\hline \multirow{3}{*}{$\begin{array}{l}\text { Ciencia, } \\
\text { tecnología y } \\
\text { sociedad }\end{array}$} & Explica la utilidad de los aparatos eléctricos & $9.8 \%$ & $28.8 \%$ & $62.2 \%$ \\
\hline & Promueve la inteligencia vial & $9.8 \%$ & $30.6 \%$ & $59.6 \%$ \\
\hline & Identifica la evolución de algún aparato & $15.5 \%$ & $39.9 \%$ & $44.6 \%$ \\
\hline \multirow[t]{2}{*}{ Entorno físico } & Explica los estados de la materia & $5.2 \%$ & $21.8 \%$ & $73.1 \%$ \\
\hline & Explica los movimientos terrestres & $17.6 \%$ & $30.6 \%$ & $51.8 \%$ \\
\hline
\end{tabular}

Fuente: Elaboración propia 2015

Los datos en las competencias genéricas; de la prueba Instrumental y sistémica muestran que la relación de las respuestas en todos los aspectos (Nunca, algunas veces, y siempre), presentan un equilibrio moderado y que la respuesta nunca es baja en relación a las otras dos. Estas dificultades en el aspecto de interpretar los temas y crear iniciativas en los estudiantes para trabajar en proyectos de vida (transversales); El docente debe mejorar su capacidad para contextualizar contenidos y enfocarlos al aprendizaje de los niños del área. Requiere del docente diseñar metodología para llevar a cabo, actividades de liderazgo y generar comportamientos importantes en los estudiantes para desempeñarse en el contexto social en el que se desenvuelven.

Los datos de las competencias docentes; en el aspecto pedagógico formar, se observa que el docente debe generar aprendizajes socialmente significativos, tener dominio $\mathrm{y}$ destrezas para enseñar $\mathrm{y}$ desarrollar habilidades de pensamiento en los niños el área de Ciencias Naturales de primaria. En la evaluación del conocimiento, debe vincular constantemente actividades de artísticas para despertar la creatividad en los niños y despertar el interés, si genera estos espacios de aprendizaje incide en la motivación hacia el área por parte de los niños de primaria.

En lo que se refiere al currículo, los estudiantes en siempre aún no alcanzan el nivel requerido en la educación referidas para este caso en Ciencias Naturales de primaria. Se Observa donde hay poca participación en la actualización en capacitaciones; Un docente debe generar espacios para capacitarse y crear dinámicas que mejoren el currículo correspondiente, es un orientador cuando apoya las acciones didácticas y formativas, donde utiliza herramientas hasta alcanzar los indicadores de desempeño en sus estudiantes de Ciencias Naturales para este caso, de primaria (Tabla II).
Enero-Diciembre 2016 ISSN 1794-8231 E-ISSN 2462 - 8794 PP: 92-100 
No. 1
Tabla II. Información Socio académica Docentes

\begin{tabular}{|c|l|c|}
\hline \multicolumn{2}{|c|}{ VARIABLE } & FRECUENCIA \\
\hline \multirow{2}{*}{ Genero } & Femenino & 15 \\
\cline { 2 - 3 } Edad & Masculino & 4 \\
\hline \multirow{3}{*}{ Antigüedad } & Máxima & 68 \\
\cline { 2 - 3 } & Mínima & 21 \\
\cline { 2 - 3 } & Media & 38 \\
\hline \multirow{2}{*}{ Horario docentes } & Máxima & 38 \\
\cline { 2 - 3 } & Mínima & 2 \\
\cline { 2 - 3 } & Media & 9 \\
\cline { 2 - 3 } & Semanal & 3 horas \\
\cline { 2 - 3 } & Anual & 120 horas \\
\hline
\end{tabular}

Fuente: Autor

De la misma forma es importante conocer las respuestas que dan los docentes en su quehacer educativo para mostrar las prácticas pedagógicas que imparten, así como el logro de competencias en sus estudiantes. Los resultados a continuación muestran una caracterización de los docentes que pertenecen al área de Ciencias Naturales en la Institución educativa de estudio teniendo presente aspectos personales, académico y profesionales.

\subsection{Edad de los Docentes}

Es importante resaltar que el promedio de edad de los docentes es de aproximadamente 38 años en general, tomadas en un rango de 21 hasta los 62 años. Las edades son importantes ya que los docentes pueden realizar posgrados y actualizaciones en relación a las competencias que requiere la institución educativa Julio Pérez Ferrero, de la ciudad de San José de Cúcuta y así avanzar en las estrategias que conducen al mejoramiento de las competencias de básica primaria y en el área de Ciencias Naturales para los grados 3, 4 y 5 grado.

\subsection{Horario Intensidad Semanal De Los Profesores}

En la Institución Educativa la intensidad semanal está estipulada por el MEN, repartida en tres horas semanales desde tercer grado hasta sexto grado, completando un total de 120 horas anuales, tiempo no suficiente para alcanzar las competencias establecidas por el MEN, especialmente, en cuanto al desarrollo del pensamiento científico que el estudiante debe lograr.

\subsection{Docentes con Pregrado}

Se puede observar en la tabla de pregrado de los maestros de la institución educativa Julio Pérez Ferrero, pertenecen al área de ciencias naturales y son mujeres; y que hace falta gestionar la ubicación de maestros en un alto porcentaje, para ciencias naturales de básica primaria; puesto que, uno de los propósitos del MEN, en su política educativa es garantizar que todos los educandos, independientemente del contexto socioeconómico y cultural del cual vienen, reciban en la escuela una educación de alta calidad que contribuya al desarrollo de competencias necesarias para vivir, convivir, ser productivos en todos los ámbitos y seguir aprendiendo a lo largo de la vida.

\subsection{Docentes con postgrado}

Se ha identificado en este cuadro un alto porcentaje de docentes que no han accedido a estudios de Postgrado, donde analicen la importancia de identificar las concepciones que deben tener los maestros sobre las competencias en el ámbito educativo que es direccionado en la Educación Superior. Y es allí donde se orienta al currículo, la docencia, el aprendizaje y la evaluación desde un marco de la calidad, fundamentalmente para dar respuesta a la demanda de las competencias genéricas y científicas de la sociedad actual, enmarcada dentro del mundo globalizante en que se vive.

La importancia de realizar estudios, por parte de los docentes, en posgrado en los campos formativos para la educación básica, en este caso para ciencias naturales; incide de manera importante en el desarrollo de las competencias científicas, Genéricas, docentes, entorno físico, entorno vivo, y ciencia, tecnología y sociedad. 


\subsection{Docentes que participan en la Institución Educativa en Proyectos Transversales}

La mitad de los docentes, no trabajan en ningún proyecto transversal, $\mathrm{y}$ menos de la mitad se sitúan en un porcentaje muy mínimo en otros proyectos, es decir, un gran porcentaje de docentes no están comprometidos en las competencias para la vida en la institución educativa Julio Pérez Ferrero. La implementación de los proyectos transversales esta soportada conceptual y metodológicamente en Normas y Políticas Nacionales claramente planteadas. Decreto 1860 de agosto 3 de 1994 (reglamenta la Ley 115 en los aspectos pedagógicos).

\section{Hallazgos}

Se resalta la importancia de estudiar las concepciones de los maestros sobre la enseñanza y sus relaciones con la práctica docente como un aspecto clave para el cambio de estas prácticas. Se puede observar en el semáforo amarillo que aún falta, para llegar a un cambio en la aplicación de las competencias específicas y genéricas por falta de más entrega por parte de los docentes, para actualizarse en capacitaciones en las nuevas pedagogías, sobre el manejo en la escuela nueva. Y apropiarse de los proyectos transversales, en los que aún no trabajan para llevar al estudiante hacia unas competencias ciudadanas de manejo prudencial. No se observó implementación de las nuevas tecnologías en el aula, que implican mayor facilidad visual en el aprendizaje de ciencias naturales de los niños de básica primaria. El manejo de las TIC es importante porque se sale de la escuela tradicional en los procesos de aprendizaje.

La investigación demostró que los resultados son confiables debido a que las preguntas de los diversos ítems son claras y utilizan un lenguaje comprensible para los niños de primaria encuestados; por presentar un criterio practico para opinar respecto a sus maestros, puesto que, para ellos es importante opinar sobre las actuaciones de sus educadores.

\section{Conclusiones}

Se observa en los resultados de las encuestas realizadas a los estudiantes de grados tercero, cuarto y quinto de primaria del colegio Julio Pérez Ferrero de la ciudad de Cúcuta, que los datos porcentuales de las competencias del docente; en lo referente al aspecto pedagógico arroja un porcentaje de respuestas algunas veces, es alto e incide directamente en las respuestas de siempre que, para este caso, son bajas. El docente debe generar aprendizajes socialmente significativos, tener dominio y destrezas para enseñar y desarrollar habilidades de pensamiento en los niños en el área de ciencias naturales de primaria. $\mathrm{Y}$ en la evaluación del conocimiento, debe vincular constantemente actividades de artísticas para despertar la creatividad en los niños y despertar el interés, si genera estos espacios de aprendizaje incide en la motivación hacia el área por parte de los niños de primaria. En los porcentajes de respuestas: algunas veces, $\mathrm{y}$ siempre, se puede analizar que existe una diferencia importante.

Elrolpedagógicoexigeunlenguajecompetente capaz de generar en los estudiantes respuestas proactivas que lo hagan salir de su cascaron y enfrentarse a nuevos retos involucrándose en el aula de clase, en las competencias generales en el área de Ciencias Naturales como lo son la interpretativa, argumentativa y propositiva. Ya que la competencia busca desarrollar la capacidad que tienen los individuos de saber aplicar lo aprehendido en la solución de las diferentes situaciones que vivencia día a día (Aprendizaje significativo). Todo esto debería ir articulado con la aplicación de nuevas estrategias didácticas que lo lleven a una aventura de aprendizajes.

Por último el docente debe recordar que cada nivel de educación (Primaria, básica y media), debe facilitar la transmisión de saberes,
Enero-Diciembre 2016 ISSN 1794-8231 E-ISSN 2462 - 8794 PP: 92-100 
acordes al contexto de los educandos, debe tener en cuenta las Competencias Ciudadanas respecto a aquellos niños, que vienen a la ciudad desplazados de la parte rural como víctimas del conflicto armado, despertar su alteridad(ponerse en el lugar del otro), porque de otra manera; estos niños estarían en desigualdad de aprendizajes, ya que más que aprendizajes cognitivos necesitan de ayuda psicológica para poder superar sus miedos (Marmolejo, 2012).

Por tal motivo, la práctica pedagógica debe situarse de acuerdo a las condiciones del contexto del educando.

\section{Referencias}

Freire. P. (s.f.). Pedagogía del oprimido. http://www.servicioskoinonia.org/biblioteca/ general/FreirePedagogiadelOprimido.pdf.

Álvarez, M., Arias, A., Pérez, U. \& Serralle, J. (2013). La historia de las ciencias en el desarrollo de competencias científicas. Enseñanza de las ciencias, 31(1), 213-233.

Bain K. (2007). Los Mejores profesores universitarios. Valencia: Universidad de Valencia. http://www.fceia.unr.edu.ar/geii/ maestria/2014/DraSanjurjo/8mas/Ken\%20 Bain, $\% 20$ Lo $\% 20$ que $\% 20$ hacen $\% 20$ los $\% 20$ mejores $\% 20$ profesores $\% 20 \mathrm{de} \% 20$ universidad.pdf

Freire, Paulo. (1997). La importancia de leer $y$ el proceso de liberación. México: Siglo XXI editores

Fullan, M. (2002). Las fuerzas del cambio. Explorando las profundidades de la reforma educativa. Madrid: Akal.

Marmolejo, G. (2012). Elementos a considerar en la enseñanza del registro semiótico de las figuras en los primeros ciclos de la educación básica. Revista Ecomatemático, 2(1), 4-19.

Boom, A.M. (2012). Práctica Pedagógica: Historia y presente de un concepto"
Práctica Pedagógica. Perspectivas Teóricas. Colombia: ECOE EDICIONES.

Méndez, D., Méndez, A. \& Fernández, J. (2015). Análisis y valoración del proceso de incorporación de las Competencias Básicas en Educación Primaria. Revista de Investigación Educativa, 33(1), 233-246.

Mora, W. \& Parga, D. (2005). Evaluación por competencias y estándares de competencia en el campo de la enseñanza de las ciencias y la educación ambiental. Enunciación, 10(1), 7384.

Torres, A. \& Barrios, A. (2009). La enseñanza de las Ciencias Naturales y educación ambiental en las instituciones educativas oficiales del departamento de Nariño. Revista de la Facultad de Ciencias Económicas y Administrativas, 10(1), 143-166.

Torres, A., Mora, E., Garzón, F. \& Ceballos, N. (2013). Desarrollo de competencias científicas a través de la aplicación de estrategias didácticas alternativas. Un enfoque a través de la enseñanza de las Ciencias Naturales. Revista de la Facultad de Ciencias Económicas y Administrativas, 15(1), 187215.

Barragán, D., Gamboa, A., \& Urbina, J.E, (2013). Práctica Pedagógica. Colombia: ECOE EDICIONES.

Pisa. (2011). PISA: Competencia Científica para el mundo del mañana. España: Instituto Vasco de Evaluación e Investigación Educativa. http://www.isei-ivei.net/cast/ pub/itemsliberados/Ciencias2011/ciencias_ PISA2009completo.pdf

CharpakG.(2004).Eldesafio delrazonamiento científico de cómo lo revolucionario no es transformar la propia enseñanza de la ciencia. Bogotá: Ministerio de Educación Nacional. http://www.mineducacion.gov. co/1621/article-87480.html. 